\title{
Broadband RF Phased Array Design for UHE neutrino detection
}

\author{
Jordan C. Hanson ${ }^{a, *}$ \\ ${ }^{a}$ Whittier College, \\ 13406 East Philadelphia Street, Los Angeles, California, 90602 \\ E-mail: jhanson2@whittier.edu
}

\begin{abstract}
Radio-frequency (RF) phased array systems have a wide variety of applications in engineering and physics research. Among these applications is ultra-high energy neutrino (UHE- $v$ ) detection above $100 \mathrm{PeV}$ via the Askaryan effect. Phased array design usually requires numerical modeling with expensive commercial computational packages. Using the open-source MIT Electrogmagnetic Equation Propagation (MEEP) package, a set of phased array designs relevant for UHE- $v$ detection is presented. Specifically, one-dimensional arrays of Yagi-Uda and horn antennas were modeled in the bandwidth of the Askaryan effect [0.1 - 5] GHz, and compared to theoretical expectations. Precise matches between MEEP simulation and radiation pattern predictions at different frequencies and beam angles are demonstrated. Finally, the effect of embedding a phased array within Antarctic ice is studied. Askaryan-class UHE- $v$ detectors are being constructed in Antarctic ice because it is an ideal detection medium for UHE- $\nu$. Future work will develop the phased array concepts with parallel MEEP, in order to increase the detail, complexity, and speed of the computations.
\end{abstract}

$37^{\text {th }}$ International Cosmic Ray Conference (ICRC 2021)

July 12 th - 23rd, 2021

Online - Berlin, Germany

\footnotetext{
${ }^{*}$ Presenter
} 


\section{Introduction}

Phased arrays have applications in 5G, ground penetrating radar (GPR), and scientific instrumentation $[13,14,16]$. A series of three-dimensional antenna elements are arranged in a line with fixed spacing [2]. Proprietary RF modeling packages like XFDTD and HFSS are often used to model phased arrays $[1,8,12]$. The XFDTD package, for example, relies on the finite difference time domain (FDTD) method. The FDTD approach is a computational electromagnetics (CEM) technique in which Maxwell's equations are solved in discrete spacetime. Two advantages of the open-source approach to RF design are zero-cost and fine control over simulation objects. Maxwell's equations are scale-invariant, so FDTD codes designed for $\mu \mathrm{m}$-wavelengths can be re-purposd for RF designs. One such package is the MIT Electromagnetic Equation Propagation (MEEP) package [15].

A recent review [7] covered open-source software facilitates RF antenna design. In this work, the radiation patterns of one-dimensional phased array designs are simulated with the MEEP package. Common MEEP applications have $\mu \mathrm{m}$-scale wavelengths, but scale-invariance allows the user to treat designs as cm-scale RF elements. Two classes of phased array element are considered: Yagi-Uda and horn antennas. The former is applied to single-frequency designs, while the latter is applied to broadband design. The phase-steering properties and radiation patterns of all designs are shown to match theoretical predictions. The appropriate array theory is shown in Section 2. Section 3 contains comparisons between theory and simulation. In Section 4, the effect of a varying index of refraction is introduced.

\section{Phased Array Antenna Theory}

The structure of a one-dimensional phased array is shown in Figure 1. Two constants that determine the beam angle $\Delta \phi$ of the array are the spacing $d_{y}$ and the phase shift per antenna $\Delta \Phi$. Letting the subscript $i$ label each of the $N$ elements, the one-dimensional spacing in Figure 1 is $d_{y} \hat{j}=\vec{r}_{i+1}-\vec{r}_{i}$. The phase shift per antenna is $\Delta \Phi=\Phi_{i+1}-\Phi_{i}$. The relationship between $d_{y}, \Delta \Phi$, and $\Delta \phi$ is quoted in Section 2.1. The radiation pattern for a given $\Delta \phi$ is quoted in Section 2.2. For all coordinate systems, the azimuthal angle in the xy-plane is $\phi$, and the polar angle from the z-axis is $\theta$.

\subsection{Phase Steering and Beam Angle}

According to [10], the beam angle $\Delta \phi$ of the array given $\Delta \Phi$ and $d_{y}$ is

$$
\Delta \Phi \approx 2 \pi\left(d_{y} / \lambda\right) \Delta \phi
$$

Equation 1 relates $\Delta \phi$, the beam angle, and $\Delta \Phi$, the phase shift per element, via a linear relationship with slope $\lambda /\left(2 \pi d_{y}\right)$.

\subsection{Radiation Patterns and Beam Width}

Let $N$ be the number of radiating elements in the phased aray. Further, let the beam angle be $\Delta \phi=\phi-\phi_{0}$, where $\phi_{0}$ is some reference angle (usually in the direction orthogonal to the phased array). According to [10], the radiation pattern is 


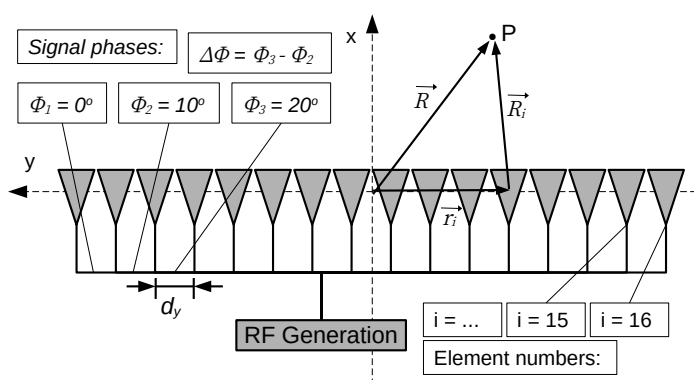

Figure 1: Definitions for the coordinate system, element label $i$, position vectors, and phase shift per antenna for a one-dimensional phased array of RF radiating elements. An example phase shift per antenna of $\Delta \Phi=\Phi_{2}-\Phi_{1}=\Phi_{3}-\Phi_{2}=\Phi_{i+1}-\Phi_{i}=10^{\circ}$ value is shown. Example position vectors for the 12th element are shown: $\vec{R}=\vec{r}_{12}+\vec{R}_{12}$.

$$
P(\phi)=\left(\frac{\sin \left(\pi N\left(d_{y} / \lambda\right)\left(\sin (\phi)-\sin \left(\phi_{0}\right)\right)\right)}{N \sin \left(\pi\left(d_{y} / \lambda\right)\left(\sin (\phi)-\sin \left(\phi_{0}\right)\right)\right)}\right)^{2}
$$

The radiation pattern in Equation 2 is shown to match precisely the main beam of FDTD calculations via MEEP for one-dimensional arrays in Section 3. All one-dimensional array radiation patterns correspond to the E-plane (the xy-plane). The MEEP python routine get_farfield is evaluated at a radius $r \gg L$, the length of the array, to obtain the far-fields $\vec{E}$ and $\vec{H}$. Notice that not all open-source FDTD codes offer near-field to far-field projection [7].

\section{Results: Phase Steering, Beam Angle, Beamwidth, and Radiation Pattern}

The two element designs are depicted in Figure 2. The Yagi-Uda antennas have 6 elements with the same radius, oriented in the xy-plane: one reflector, one radiatior, three directors and a connecting boom. The horn antennas have three structures: the box containing the linearly polarized radiator, the radiator, and the curves of the horn. An exponential function describes the curves. All antenna elements have metallic conductivity, and the edge of space consists of a perfectly matched layer (PML) to cancel reflections.

\subsection{Phase Steering, Beam Angle, and Beamwidth}

Phase steering, beam angle, and beamwidth results are shown in Figure 3. The y-axes of Figure 3 (top left) and (top right) are the beam angles of the Yagi-Uda arrays, divided by the beam widths. The $\mathrm{x}$-axes are the phase shifts per element. The top left and top right plots corresond to $N=8$ and $N=16$, respectively. For the $N=16$ horn case (bottom left and right), the value of $d_{y} / \lambda=f d_{y} / c$ varies because the elements can radiate from $\approx 0.3-5.0 \mathrm{GHz}$. The black solid lines in the top left and top right graphs of Figure 3 are linear fits to the Yagi-Uda data. The gray lines represent the function $f(x)=b x$, with $b=\lambda /\left(2 \pi d_{y}\right)$. The slopes match, with $1 \%$ errors arising from pattern distortion for large $\Delta \phi$. At large $\Delta \Phi$, side lobes can merge with the main beam to shift the location of the main beam by $O(1)$ degree.

For the broadband horn case in the bottom left of Figure 3, data from three frequencies are shown: $0.3,1.5$, and $3.0 \mathrm{GHz}$. The intercepts are all zero and the slopes scale correctly. The fit 

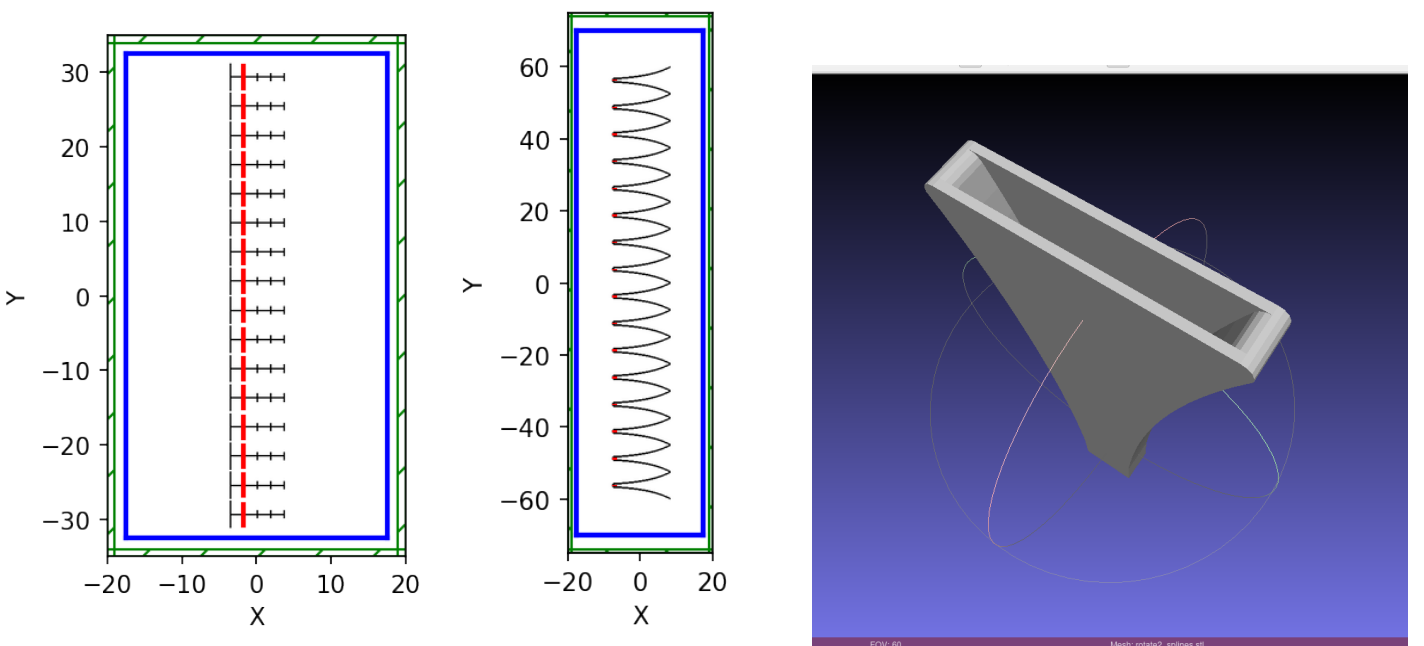

Figure 2: The 2D antenna designs used in the $1 \mathrm{D}$ phased array simulations. (Left) The $N=16$ Yagi-Uda array. (Middle) The $N=16$ horn array. The blue surfaces are MEEP Near2FarRegion objects for near-to-far projection. All dimensions are in centimeters. (Right) Example STL file for 3D version of horn.
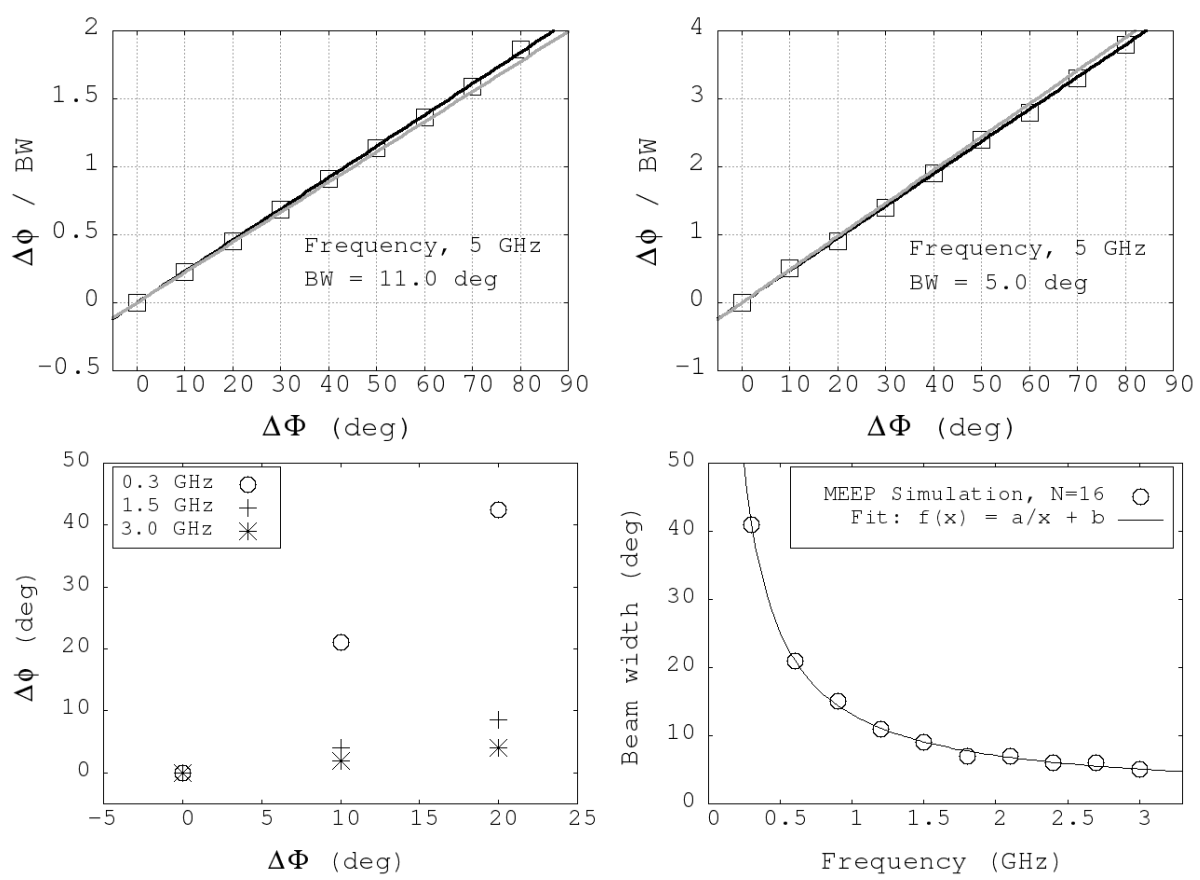

Figure 3: (Top left) The beam angle $\Delta \phi$ divided by the beam width $B W$ for the $N=8$ one-dimensional Yagi array versus $\Delta \Phi$, the phase shift per element. (Top right) The same results for the $N=16$ array. (Bottom left) $\Delta \phi$ versus $\Delta \Phi$ for the $N=16$ version of the one-dimensional horn array, for several frequencies. (Bottom right) The dependence of the beam width on frequency for the one-dimensional $N=16$ horn array. 

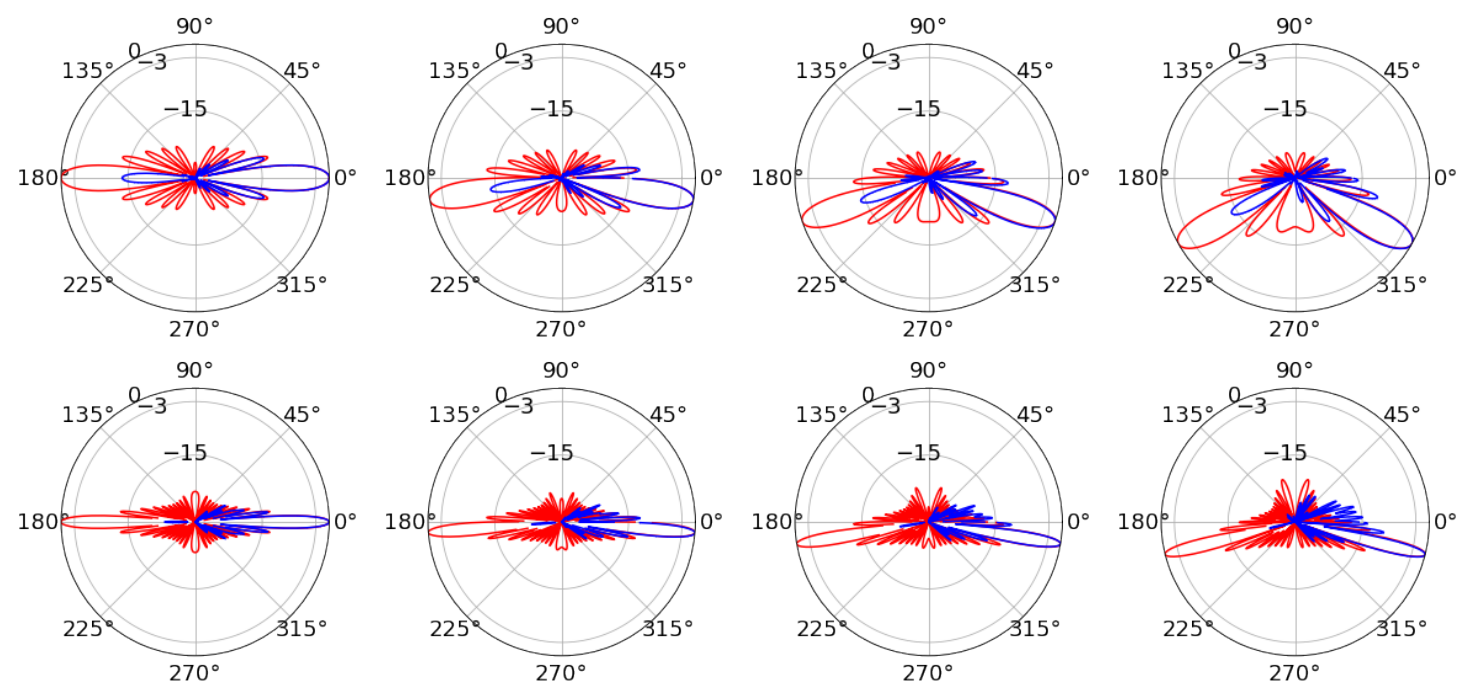

Figure 4: Yagi-Uda results. (Top row) $f=2.5 \mathrm{GHz}$, and $\Delta \Phi=0,20,40,60$ degrees from left to right. (Second row) $f=5.0 \mathrm{GHz}$, and $\Delta \Phi=0,20,40,60$ degrees from left to right. The radial units are $\mathrm{dB}$, and the angular units are degrees.

parameters for beam width $f(x)=a / x+b$ were $a=12.0 \pm 0.1$ degree $\mathrm{GHz}$, and $b=1.1 \pm 0.2$ degrees. The $1 / f$-dependence is a good description of the beamwidth across the $[0.3-5 \mathrm{GHz}]$ bandwidth. The constant term $b$ is only necessary since the array has finite length $L$.

\subsection{Radiation Patterns}

Radiation patterns for $N=16$ one-dimensional arrays are shown in Figures 4 and 5, respectively. As described above, the x-direction $(\Delta \phi=0)$ corresponds to no phase shift per element $(\Delta \Phi=0)$. The radiation patterns are shown in blue, and the red curves represent Equation 2. Equation 2 is symmetric, with identical forward and backward lobes.

The Yagi-Uda results are shown for 2.5 and $5.0 \mathrm{GHz}$ frequencies in Figure 4, with $\Delta \Phi=$ $0,20,40$, and 60 degrees. The amplitudes of all side-lobes are limited to $\approx-15 \mathrm{~dB}$, except at the highest beam angles where scan losses are experienced. The effect of frequency on beam steering is evident. The same $\Delta \Phi$ does not generate as large a $\Delta \phi$ at higher frequencies because the slope implied by Equation 1 is proportional to $\lambda$.

The horn results are shown in Figure 5 for $0.5 \mathrm{GHz}$ and $5.0 \mathrm{GHz}$ frequencies. The phase shifts per element are $\Delta \Phi=0,10,20$, and 30 degrees. Wideband systems experience a natural trade-off in angular range versus bandwidth. A $d_{y} / \lambda$ value that is acceptably smaller than one at low frequencies can grow larger with increasing frequency, leading to interference patterns. At 5.0 $\mathrm{GHz}$, the horns radiate at \pm 45 degrees from $\Delta \phi=0$. The prediction from Equation 2 is that these grating lobes are equal in relative power to the main beam. The horns limit them to $-15 \mathrm{~dB}$ if $|\Delta \Phi|<35$ degrees. 

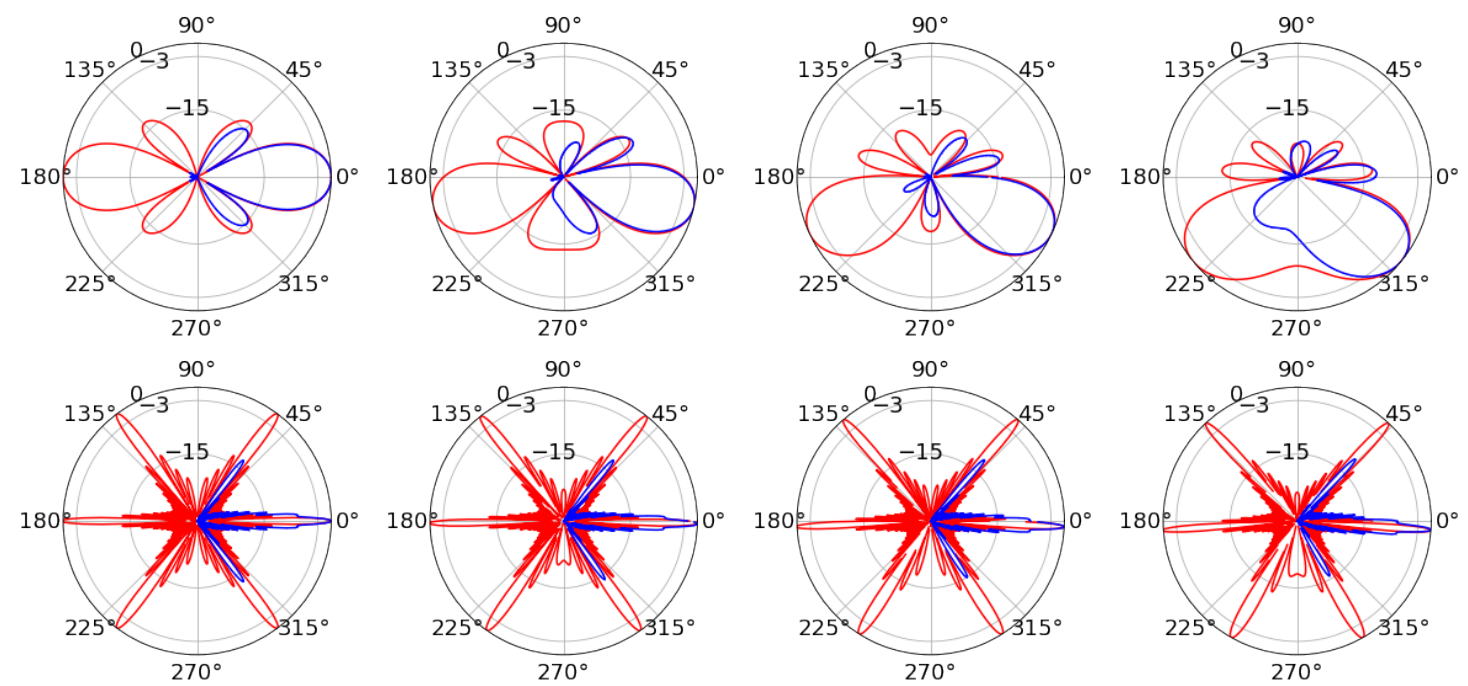

Figure 5: Horn results. (Top row) $f=0.5 \mathrm{GHz}$, and $\Delta \Phi=0,10,20,30$ degrees from left to right. (Second row) $f=5.0 \mathrm{GHz}$, and $\Delta \Phi=0,10,20,30$ degrees from left to right. The radial units are $\mathrm{dB}$, and the angular units are degrees.

\section{Variation of the Index of Refraction}

The behavior of a phased array embedded within a spatially-dependent index of refraction $n(z)$ is interesting to the UHE- $v$ community $[2,16]$. Phased arrays represent an opportunity to lower the RF detection threshold for RF pulses generated by UHE- $v$ via the Askaryan effect. Ice is the most convenient medium for Askaryan pulse detection due to the RF transparency and large pristine volumes located in Antarctic and Greenlandic ice sheets and shelves [3, 9, 11]. The index of refraction varies within the ice because of the transition between surface snow $\left(\rho \approx 0.4 \mathrm{~g} / \mathrm{cm}^{3}\right)$ and the solid ice below $\left(\rho=0.917 \mathrm{~g} / \mathrm{cm}^{3}\right.$ ). Embedded phased arrays with varying $n(z)$ emit signals that curve in the direction of increasing $n(z)$.

The shadow zone is the volume of ice from which RF signals do not reach a receiver due to the excess curvature of the ray trace [6]. While there is evidence that RF signals can propagate horizontally through Antarctic ice [4], data from Greenland suggests the relative strength of the effect is small compared to the curved radiation [5]. It is possible to map out the shadow zone for an embedded phased array radiating at fixed frequency. Grating lobe power reflects downward from the snow-air interface, and radiates into the shadow zone. Grating lobe power also refracts into the air above the interface.

A two-parameter fit to the $n(z)$ data versus depth $z$ below the surface is given by [4]

$$
n(z)= \begin{cases}1 & z>0 \\ n_{\text {ice }}-\Delta n \exp \left(z / z_{0}\right) & z \leq 0\end{cases}
$$

The fit parameters in Equation 3 come from Reference [4]: $\Delta n=0.423 \pm 0.004$ and $z_{0}=77 \pm 2$ meters, with $n_{\text {ice }}=1.78$. These values are derived from the SPICE core near the South Pole. Equation 3 was implemented in a volume surrounding a horn array with all but the radiators removed. Further, the length scale was reinterpreted to be meters rather than centimeters. The $\lambda / 4$ 
dipoles were spaced by $\lambda / 2$ according to their free space $\lambda$ value. At $200 \mathrm{MHz}$, the dipole length is 0.375 meters, and the spacing is 0.75 meters.

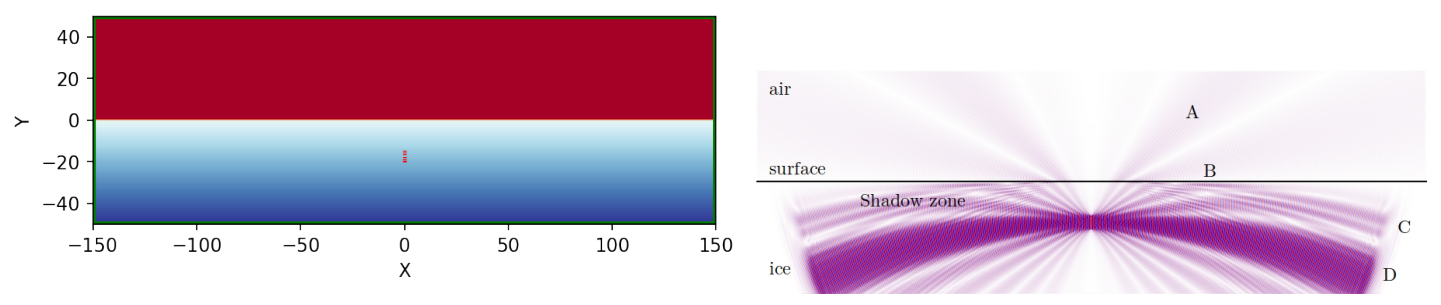

Figure 6: (Left) The simplified $N=8$ phased array with dimensions in meters. The colorscale represents $n(z)$ in Equation 3. (Right) The magnitude of the z-component of the E-field from the vertical dipoles. The air, surface, and ice regions are the same as (left). The array depth is -15 meters. (A) Radiation refracting through the surface into the air. (B) Grating lobes reflect from the surface into the shadow zone. (C) Grating lobes propagating through the shadow zone. (D) The main beam bent downward due to the gradient in $n(z)$.

Figure 6 contains the results of a $N=8$ dipole array embedded in a medium with the index profile in Equation 3. Figure 6 (left) shows the schematic of the calculation, and Figure 6 (right) shows the magnitude of the z-component of the radiated E-field. The units in Figure 6 are meters, and the radiation frequency is $200 \mathrm{MHz}$. The distance between the air-snow interface and the first phased array element is 15 meters. The color scale in Figure 6 (right) is \pm 0.05 with the signal amplitude of the elements \pm 1.0 at $200 \mathrm{MHz}$. The amplitude scale is less important than observing where the radiation has penetrated the ice after 200 radiation periods. In Figure 6 (right), the main beam has curved downwards in the direction of increasing $n(z)$, while grating lobes have both diffracted to the air and reflected into the shadow zone. The rate of curvature of the main beam is controlled by the fit parameter $z_{0}$ in Equation 3 .

\section{References}

[1] Ansys, Inc., Canonsburg, Pennsylvania. 3D Electromagnetic Field Simulator for RF and Wireless Design, 2020.

[2] J. Avva, K. Bechtol, T. Chesebro, L. Cremonesi, C. Deaconu, A. Gupta, A. Ludwig, W. Messino, C. Miki, R. Nichol, E. Oberla, M. Ransom, A. Romero-Wolf, D. Saltzberg, C. Schlupf, N. Shipp, G. Varner, A. Vieregg, and S. Wissel. Development toward a groundbased interferometric phased array for radio detection of high energy neutrinos. Nuclear Instruments and Methods in Physics Research Section A: Accelerators, Spectrometers, Detectors and Associated Equipment, 869:46 - 55, 2017.

[3] J. Avva, J. Kovac, C. Miki, D. Saltzberg, and A. Vieregg. An in situ measurement of the radio-frequency attenuation in ice at Summit Station, Greenland. Journal of Glaciology, 2014.

[4] S. W. Barwick, E. C. Berg, D. Z. Besson, G. Gaswint, C. Glaser, A. Hallgren, J. C. Hanson, S. R. Klein, S. Kleinfelder, L. Köpke, I. Kravchenko, R. Lahmann, U. Latif, J. Nam, A. Nelles, C. Persichilli, P. Sandstrom, J. Tatar, and E. Unger. Observation of classically ‘forbidden' electromagnetic wave propagation and implications for neutrino detection. Journal of Cosmology and Astroparticle Physics, 2018(07):055-055, 2018. 
[5] C. Deaconu, A. G. Vieregg, S. A. Wissel, J. Bowen, S. Chipman, A. Gupta, C. Miki, R. J. Nichol, and D. Saltzberg. Measurements and modeling of near-surface radio propagation in glacial ice and implications for neutrino experiments. Phys. Rev. D, 98:043010, Aug 2018.

[6] K. Dookayka. Characterizing the Search for Ultra-High Energy Neutrinos with the ARIANNA Detector. PhD thesis, Univeristy of California at Irvine, 2011.

[7] A. Fedeli, C. Montecucco, and G. L. Gragnani. Open-Source Software for Electromagnetic Scattering Simulation: The Case of Antenna Design. Electronics, 8(12):1506, 2019.

[8] N. Feng, Y. Zhang, X. Tian, J. Zhu, W. T. Joines, and G. P. Wang. System-Combined ADIFDTD Method and Its Electromagnetic Applications in Microwave Circuits and Antennas. IEEE Transactions on Microwave Theory and Techniques, 67(8):3260-3270, 2019.

[9] J. C. Hanson. Ross Ice Shelf Thickness, Radio-frequency Attenuation and Reflectivity: Implications for the ARIANNA UHE Neutrino Detector. In Proceedings of the 32nd International Cosmic Ray Conference, Beijing, China, August 2011, 2011.

[10] J. C. Hanson. Broadband rf phased array design with meep: Comparisons to array theory in two and three dimensions. Electronics, 10(4), 2021.

[11] J. C. Hanson, S. W. Barwick, E. C. Berg, D. Z. Besson, T. J. Duffin, S. R. Klein, S. A. Kleinfelder, C. Reed, M. Roumi, T. Stezelberger, J. Tatar, J. A. Walker, and L. Zou. Radar absorption, basal reflection, thickness and polarization measurements from the Ross Ice Shelf, Antarctica. Journal of Glaciology, 61(227):438-446, 2015.

[12] T. Q. Ho, L. N. Hunt, C. A. Hewett, T. G. Ready, R. Mittra, W. Yu, D. A. Zolnick, and M. Kragalott. Analysis of Electrically Large Patch Phased Arrays via CFDTD. 2006 IEEE Antennas and Propagation Society International Symposium, Albuquerque, New Mexico, July 2006.

[13] K. Kikuchi, H. Mikada, and J. Takekawa. Improved Imaging Capability of Phased Array Antenna in Ground Penetrating Radar Survey. Conference Proceedings, 79th EAGE Conference and Exhibition 2017, Paris, France, June 2017, 2017.

[14] T. Munekata, M. Yamamoto, and T. Nojima. A Wideband 16-Element Antenna Array Using Leaf-Shaped Bowtie Antenna and Series-Parallel Feed Networks. 2014 IEEE International Workshop on Electromagnetics (iWEM), Sapporo Hokkaido, Japan, August 2014, pages 8081, 2014.

[15] A. F. Oskooi, D. Roundy, M. Ibanescu, P. Bermel, J. Joannopoulos, and S. G. Johnson. Meep: A flexible free-software package for electromagnetic simulations by the FDTD method. Computer Physics Communications, 181(3):687-702, 2010.

[16] A. Vieregg, K. Bechtol, and A. Romero-Wolf. A technique for detection of PeV neutrinos using a phased radio array. Journal of Cosmology and Astroparticle Physics, 2016(02):005, feb 2016. 